\title{
Empleo del NDVI de una camara digital modificada para estimar la cobertura del cultivo de papa bajo distintas condiciones de fertilización nitrogenada
}

\author{
de la Casa, A.; G. Ovando, L. Bressanini y J. Martínez
}

\begin{abstract}
RESUMEN
La fracción del suelo cubierta por el cultivo (CC) determina tanto la intercepción de radiación solar como el tamaño del aparato transpiratorio. Asimismo, la disponibilidad de nitrógeno (N) en papa (Solanum tuberosum L.) afecta el rendimiento y la calidad de los tubérculos. Este trabajo desarrolla procedimientos digitales para estimar la CC en papa usando imágenes fotográficas de cámaras convencionales y modificadas para obtener datos del índice de vegetación de la diferencia normalizada (NDVI), evaluados bajo distintas condiciones de fertilización nitrogenada en cultivos de ciclo tardío en 2012 y 2013. En 2012 se comparó la CC y rendimiento de un sector que no recibió fertilización a la plantación respecto del tratamiento comercial, en tanto que en 2013 se consideraron cuatro tratamientos con distintas dosis de N. Ambas cámaras, como así también los procedimientos para estimar la CC, resultaron apropiados para acusar diferencias significativas en la CC asociadas a la fertilización en 2012. Mientras la menor fertilización en 2012 produjo una disminución anticipada de la CC al final del ciclo, lo que redujo la productividad y la calidad comercial, en 2013 las diferencias de la CC y rendimiento entre tratamientos fueron menos pronunciadas.
\end{abstract}

Palabras clave: Solanum tuberosum L., cámara digital, fertilización, teledetección.

de la Casa, A.; G. Ovando, L. Bressanini and J. Martínez, 2016. Using NDVI from a modified digital camera to estimate potato crop cover under different conditions of nitrogen fertilization. Agriscientia 33 (2): 75-88

\section{SUMMARY}

The fraction of the ground covered by a crop (CC) determines both interception of solar radiation and the size of the transpiration apparatus. Besides, the 
availability of nitrogen $(\mathrm{N})$ in the potato crop (Solanum tuberosum L.) affects the yield and the quality of tubers. Digital procedures for estimating CC in potato from conventional and modified cameras to calculate Normalized Difference Vegetation Index (NDVI) were developed and evaluated under different conditions of nitrogen fertilization in potato commercial fields during the late growing season of 2012 and 2013. In 2012, the CC and the yield of a sector that received no fertilizer at planting were compared to the commercial treatment, while in 2013 four treatments with different doses of $\mathrm{N}$ were considered. Both cameras, as well as the procedures for estimating CC, rendered appropriate results to indicate significant differences in $\mathrm{CC}$ related to fertilization status in 2012. While the lower fertilization in 2012 produced an early decrease of CC at the end of the growing season reducing both productivity and commercial quality of tubers, in 2013 the CC and yield differences between treatments were less significant.

Key words: Solanum tuberosum L., digital camera, fertilization, remote sensing.

A. de la Casa, G. Ovando, L. Bressanini y J. Martínez. Facultad de Ciencias Agropecuarias, Universidad Nacional de Córdoba. Correspondencia a: delacasa@agro.unc.edu.ar

\section{INTRODUCCIÓN}

La fracción del suelo cubierta por el follaje o cobertura del cultivo (CC), es decir la proporción de la superficie del terreno que es cubierta por el canopeo verde visto desde arriba, constituye un parámetro biofísico utilizado para representar distintas funciones del crecimiento y desarrollo de los cultivos. La CC se emplea, en este sentido, para determinar tanto la tasa radiación solar que intercepta el canopeo de un cultivo (de la Casa et al., 2007; 2012), como para representar el tamaño del aparato transpiratorio y, a partir del concepto de productividad del agua, estimar el rendimiento (de la Casa et al., 2013).

El mercado de comercialización de la papa (Solanum tuberosum L.) está poniendo énfasis de manera creciente en la calidad del producto cosechado, tanto si se lo destina a la industria como al consumo doméstico. En este sentido, el productor no sólo necesita obtener rendimientos elevados sino también tubérculos de buena calidad (Steduto et al., 2009). El nitrógeno (N) es el nutriente que mayor atención ha recibido en el cultivo de papa, porque tanto su deficiencia como exceso pueden perjudicar el crecimiento y desarrollo del cultivo en términos de rendimiento y calidad del producto cosechado (Caldiz, 2006; Giletto et al., 2010).

A través del registro de la energía que reflejan y emiten los objetos, la teledetección constituye una técnica ampliamente difundida para detectar la presencia de vegetación, como así también para determinar distintos parámetros biofísicos, incluyendo la CC (Hatfield et al., 2008). La reflectancia espectral de un cultivo se correlaciona con el crecimiento alcanzado y la biomasa producida ( $\mathrm{Li}$ et al., 2010), y ha demostrado potencial también a los efectos de proporcionar información sobre el contenido de N de la vegetación (Raun et al., 2008).

El análisis a partir de imágenes digitales se ha aplicado de manera creciente en diferentes campos de investigación agronómica: evaluación de la porosidad de barreras rompevientos (Kenney, 1987), determinación de la diferencia de color y fertilidad en cultivos de maíz (Ewing \& Horton, 1999), estimación de la fracción de suelo cubierto por el canopeo de soja (Purcell, 2000), o cuantificación del número de flores de un árbol (Adamsen et al., 2000), entre otras aplicaciones.

Si bien existen diferentes procedimientos para la estimación remota de CC, la técnica de correlación y regresión a partir de indicadores espectrales ha sido la más difundida, y el índice de vegetación de la diferencia normalizada (NDVI), uno de los más empleados (Johnson \& Trout, 2012; Jia et al., 2016). La primera generación de sensores multiespectrales de la vegetación (Landsat 5 TM) ya disponía de cuatro bandas relativamente anchas en los rangos del visible e infrarrojo cercano para evaluar la presencia y productividad de los 
cultivos a partir de la relación de la reflectancia de estas bandas, porque, en principio, con ellas es posible distinguir entre los sectores cubiertos con vegetación del suelo desnudo (Rouse et al., 1973). No obstante, Adamsen et al. (1999) utilizaron una cámara digital a color para determinar el verdor del follaje en trigo realizando el cálculo de la relación entre la banda verde $(v)$ y la banda roja $(r)$ de cada píxel de la imagen, o sea con información espectral exclusivamente del rango visible. También Gitelson et al. (2002) desarrollaron un indicador denominado índice del visible atmosféricamente resistente (VARI), que utiliza exclusivamente datos radiométricos en el rango del visible para estimar la condición de cobertura en maíz y trigo.

Hunt et al. (2005) sostienen que debido a las diferencias espectrales entre la vegetación y el suelo en las longitudes de onda del verde y del rojo, las imágenes a color de cámaras digitales pueden ser útiles para determinar tanto la biomasa producida como el estado nutricional de los cultivos, de modo que emplearon el índice de la diferencia verde-rojo normalizada (NGRDI) para tener en cuenta las diferencias en la exposición (cantidad de luz que recibe el sensor de imagen). Sin embargo, mientras el NGRDI se correlacionó con la biomasa de distintos cultivos entre 0 y $120 \mathrm{~g} \mathrm{~m}^{-2}$, perdió sensibilidad cuando el valor de biomasa superó $150 \mathrm{~g} \mathrm{~m}^{-2}$. El NGRDI tampoco mostró diferencias en el estado de $\mathrm{N}$ de un experimento de fertilización en maíz, aunque las áreas de bajo contenido en $\mathrm{N}$ fueron evidentes en fotografías digitales al final del ciclo.

$\mathrm{Li}$ et al. (2010) emplearon datos de reflectancia espectral en el rango visible ( $v$ y $r$ ) para elaborar indicadores de estimación de la cobertura del follaje en trigo, y establecieron que estos valores estuvieron estrechamente correlacionados con los valores de NDVI obtenidos a partir de los dispositivos comerciales GreenSeeker ${ }^{\circledR}$ y Yara N-Sensor. Estos últimos utilizan la reflectancia del rojo e infrarrojo cercano de la cubierta del cultivo. Asimismo, determinaron que las estimaciones obtenidas a partir de una cámara digital comercial o a partir de sensores más especializados proporcionan información equivalente sobre la condición de fertilización de un cultivo.

En tal sentido Jia et al. (2016) monitorearon el crecimiento y contenido de $\mathrm{N}$ de un cultivo de algodón y obtuvieron buenas correlaciones entre la CC estimada a partir de la cámara digital y el NDVI proveniente de GreenSeeker, contenido de nitrógeno total aéreo, índice de área foliar y biomasa aérea. Meyer \& Neto (2008) emplearon tres índices de vegetación basados en los contadores digitales (0 a 255) de los canales RGB $-r, v$ y azul (a)- de fotografías digitales para clasificar y separar plan- tas individuales del entorno. Hunt et al. (2010) utilizaron un índice de vegetación calculado a partir de los contadores digitales de los canales verde e infrarrojo cercano obtenidos de una cámara digital montada sobre un vehículo aéreo no tripulado (UAV), para monitorear el índice de área foliar de un cultivo de trigo en dos lotes con fertilizaciones variables. Jannoura et al. (2015) emplearon los contadores digitales de las bandas $r y \mathrm{v}$ de una cámara digital para calcular un índice de vegetación que relacionaron linealmente con la materia seca de la parte aérea de diferentes cultivos.

En vistas de la creciente accesibilidad a los recursos digitales, ya sea a partir de cámaras convencionales sensibles sólo a la parte visible del espectro, como de dispositivos que se modifican incorporando información del infrarrojo, el objetivo de este trabajo fue desarrollar y evaluar procedimientos digitales destinados a estimar la cobertura en el cultivo de papa en distintas condiciones de fertilización nitrogenada, de un modo simple, no destructivo, e igual de efectivo que otros métodos de mayor costo económico y operativo.

\section{MATERIALES Y MÉTODOS}

\section{Determinación de la cobertura del cultivo (CC)}

La CC se determinó a partir del análisis de fotografías digitales obtenidas en forma vertical a $2 \mathrm{~m}$ de altura sobre el cultivo de papa. Las fotografías fueron tomadas aproximadamente en el mismo lugar del ensayo, en distintas fechas, durante el ciclo de cultivo, utilizando de manera alternativa dos cámaras fotográficas distintas. Se empleó una cámara convencional marca CyberShoot (Sony) y otra modificada marca Powershot SD780 IS (Canon), que posee un filtro para obtener registros en el rango del infrarrojo cercano y, de esta forma, calcular el NDVI. Ambas cámaras se configuraron para producir imágenes de baja resolución (VGA, 640 pixeles de ancho por 480 de alto) para agilizar el procesamiento y se almacenaron en formato JPEG para su procesamiento posterior.

La obtención de la CC en papa se realizó de acuerdo a los siguientes procedimientos:

\section{Método de máxima verosimilitud (MMV)}

Este procedimiento efectúa la clasificación supervisada de la imagen obtenida por una cámara convencional siguiendo el método de máxima verosimilitud (Eastman et al., 2005). El algoritmo que se desarrolló a tal efecto, prevé la identificación de 
cuatro clases distintas: suelo y follaje, al sol y en penumbra, respectivamente, de modo que la técnica requiere delimitar sobre la imagen un conjunto de píxeles para cada una de estas condiciones. A partir de los valores medios y de desviación estándar de los contadores digitales de las muestras seleccionadas sobre la pantalla, el algoritmo asigna cada píxel a su respectiva categoría con base en la mayor probabilidad de pertenencia calculada. El control de los resultados se realizó en cada caso haciendo una comparación visual entre la fotografía original y la imagen clasificada. En razón de que este procedimiento ha sido usado extensivamente en teledetección como método estándar de clasificación de imágenes y, además, exige determinar visualmente una muestra de cada clase identificada, sus resultados son considerados como referencia.

\section{Método del cociente $\mathrm{v} / \mathrm{r}$ (MC)}

Utilizando la cámara convencional y, de acuerdo con Adamsen et al., (1999) y Gitelson et al., (2002), la presencia de vegetación sobre el suelo se puede establecer apelando a la descomposición colorimétrica de la imagen en el rango del visible, y efectuando en cada píxel un cociente entre los contadores digitales de las bandas $\mathrm{V} / \mathrm{r}(\mathrm{Li}$ et al., 2010). El límite para asignar la pertenencia de cada píxel se estableció en 1,05, considerando que corresponde a vegetación cuando el valor de v/r supera dicho umbral (de la Casa et al., 2012).

\section{Método del NDVI (MND)}

La cámara Canon fue modificada para obtener directamente imágenes NDVI. Procesando contadores digitales del infrarrojo cercano (nir) y el rojo (r) del espectro electromagnético, la determinación tradicional de NDVI usa la fórmula:

$$
N D V I=\frac{(n i r-r)}{(n i r+r)}
$$

Las cámaras digitales permiten la obtención por separado de las distintas bandas del espectro, de modo que una vez aislada la banda nir a través de un filtro específico y, localizada esta señal en una de las bandas de color, se puede proceder a obtener un registro del tipo NDVI. El modelo de cámara que se utilizó para este propósito registra el canal azul (a) y el canal verde ( $v$ ) de la luz visible, mientras que el rojo corresponde al nir. De esta forma, la fórmula del NDVI puede ser reescrita como:

$$
N D V I=\frac{(n i r-a)}{(n i r+a)}
$$

Mayores detalles técnicos de esta cámara modificada pueden consultarse en von Bueren et al. (2015). Respecto al empleo de distintas bandas para elaborar el índice de vegetación, Wang et al. (2007) compararon valores de NDVI calculados a partir de un radiómetro en un lote con arroz, con valores de NDVI calculados reemplazando la banda roja por la azul (BNDVI) y por la verde (GNDVI), y encontraron valores de correlación de 0,981412 entre BNDVI y GNDVI y 0,986607 entre BNDVI y NDVI.

Como en el caso anterior, la obtención de la CC requiere discriminar cada píxel de la imagen JPG entre suelo o vegetación y, a partir de la imagen binaria producida, determinar la relación entre la cantidad de pixeles con vegetación con respecto al total, expresada como fracción (0-1) o porcentaje (0-100\%). El valor utilizado como umbral en este caso fue el cero (el valor negativo se asigna a la clase suelo).

\section{Ensayos de fertilización a campo}

Con el propósito de obtener la información de CC, como así también para evaluar el efecto de la fertilización sobre la productividad, se establecieron en 2012 y 2013 dos ensayos sobre cultivos de papa de ciclo tardío, en sendos lotes del cinturón verde de la ciudad de Córdoba.

\section{Año 2012}

El ensayo de este año se realizó en un lote de

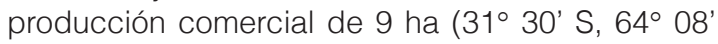
O, 402 m s.n.m.), que fue plantado el 14/02 y se cosechó el 24/05.

Las fotografías utilizadas para estimar la CC se obtuvieron de una grilla de $3 \times 3$ sectores equidistantes. Las filas, orientadas de este a oeste, corresponden a la dirección de los surcos siguiendo la pendiente natural del terreno a los efectos del riego. Sobre la intersección de cada fila con la cabecera, en una posición media y hacia el fondo, quedaron distribuidos los nueve sectores para realizar las mediciones en todo el lote.

La plantación de las 12 líneas hacia el sur de la primera fila se realizó sin fertilización (tratamiento $\mathrm{NF}$ ), en tanto las líneas siguientes hacia el norte recibieron el tratamiento de fertilización comercial con $200 \mathrm{~kg} \mathrm{ha}^{-1}$ de Yaramida (19-16-0 0,7) y 200 $\mathrm{kg} \mathrm{ha}^{-1}$ de urea (46-0-0) (tratamiento F). El 11/03 
se volvió a fertilizar todo el lote con $250 \mathrm{~kg} \mathrm{ha}^{-1}$ de urea (46-0-0). En los sectores 1.1, 1.2 y 1.3 (final del surco, posición media y cabecera, respectivamente) de la primera fila las mediciones se repitieron tres veces en distintos lugares (hacia el este, centro y oeste) para la obtención de la CC y cuatro para determinar el rendimiento final para cada tratamiento. En el resto del lote se tomaron fotografías hacia el sur y el norte de cada sector $(2.1,2.2,2.3$, 3.1, 3.2 y 3.3), para totalizar un tamaño muestral de 30 .

El rendimiento final se expresó en peso fresco y la cantidad total de tubérculos cosechados se clasificaron por tamaño en: grandes (>150 g), medianos (entre 150 y $80 \mathrm{~g}$ ) y chicos (<80 g). La evaluación estadística se realizó haciendo la prueba de $t$ para los valores medios de dos muestras apareadas, de acuerdo a la configuración del ensayo.

\section{Año 2013}

En esta oportunidad el ensayo se realizó en un lote de papa de producción comercial de 7 ha $\left(31^{\circ}\right.$ 33' S, 64 08' O, 402 m s.n.m.), que se plantó el 11/02 y cosechó el 30/05, en surcos orientados de este a oeste. Previo a la plantación se realizó un análisis del suelo en cuatro sectores del lote a partir de muestras compuestas, lo que permitió determinar condiciones edáficas similares a las de 2012. Sobre la cabecera de riego del lote se establecieron cuatro parcelas de ensayo, de ocho surcos de $28 \mathrm{~m}$ de largo, distanciados entre sí 0,85 $\mathrm{m}$, a los efectos de evaluar diferentes condiciones de fertilización. Sobre el cuarto surco central de cada parcela se distribuyeron siete estacas distanciadas a $4 \mathrm{~m}$ con el fin de realizar el seguimiento fotográfico a lo largo del ciclo, efectuando una toma por estaca aproximadamente sobre la misma superficie de terreno.

Todo el lote fue fertilizado a la plantación con $290 \mathrm{~kg} \mathrm{ha}^{-1}$ de fosfato monoamónico (11-52-0) y el 10/03 se refertilizó con $155 \mathrm{~kg} \mathrm{ha}^{-1}$ de nitro doble (27-0-0). Al día siguiente, de acuerdo a los resultados del análisis de suelo del 8/02, se establecieron cuatro tratamientos de fertilización: comercial (sin agregado de $\mathrm{N}$ adicional), y con agregado adicional de 50, 100 y $150 \mathrm{~kg} \mathrm{ha}^{-1}$ de $\mathrm{N}$ a partir de urea (46-0-0), respectivamente. En cuatro oportunidades durante el ciclo se realizó un muestreo de suelo en tres sectores de cada parcela a los efectos de determinar el contenido de N. La producción de papa se evaluó de la misma manera que el año anterior.

\section{RESULTADOS Y DISCUSIÓN}

\section{Año 2012 \\ Estimación de la cobertura}

A partir de las imágenes fotográficas extraídas extensivamente en todo el lote de papa durante este año, los resultados de la Figura 1 (A) revelan que las determinaciones de la CC de ambos procedimientos tienden a ser similares en la medida que convergen sobre la función identidad, con valores de $R^{2}$ que para cada fecha individualmente son superiores a 0,92. Al final del ciclo (7/05), cuando comienza a ocurrir la senescencia del cultivo, los valores de la CC estimados a partir de MC y MMV resultan marcadamente diferentes, debido a que el método de MC considera a los píxeles de hojas amarillentas como suelo. Un comportamiento similar fue reportado por Gitelson et al. (2014), quienes mostraron una relación entre el contenido de clorofila de maíz y el índice de área foliar que exhibe histéresis a lo largo de la estación de crecimiento del cultivo. De manera similar, Muraoka et al. (2012) también presentaron una relación con histéresis entre LAI y NDVI de bosques caducifolios de hoja ancha en Japón. Excluyendo esta fecha del análisis, la función de ajuste entre la CC estimada por ambos procedimientos es $y=0,9598 x$ $+2,223$, con un $R^{2}=0,989(P<0,001)$, alcanzando menor dispersión en general que cuando la relación se establece a partir de las estimaciones de MND (Figura 1, B).

La Figura 1 (B) muestra que los procedimientos desarrollados a partir de los registros de ambas cámaras fotográficas producen estimaciones de la CC que igualmente se presentan similares, aunque con una ligera y sistemática sobrestimación cuando la determinación se realiza a partir de MND. Al considerar el conjunto completo de datos, la función de ajuste $y=1,0315 x+3,9423$ alcanza un coeficiente de determinación $\left(R^{2}\right)$ de 0,956 $(P<$ 0,001 ), aunque en cada fecha individualmente la dispersión es más marcada, como indican en cada caso los $R^{2}$ más bajos de la Figura 1 (B). En este sentido, la menor dispersión que se produce entre las estimaciones de MC y MMV en cada fecha se justifica, ya que los resultados, si bien se obtienen de procedimientos de cálculo distintos, corresponden al procesamiento de la misma imagen, mientras que en el análisis entre MND y MMV (Figura 1 abajo) no sólo cambian los métodos para obtener $\mathrm{CC}$, sino también las cámaras digitales y, en consecuencia, las imágenes procesadas proceden de sectores que no se superponen exactamente.

De manera similar al comportamiento obser- 


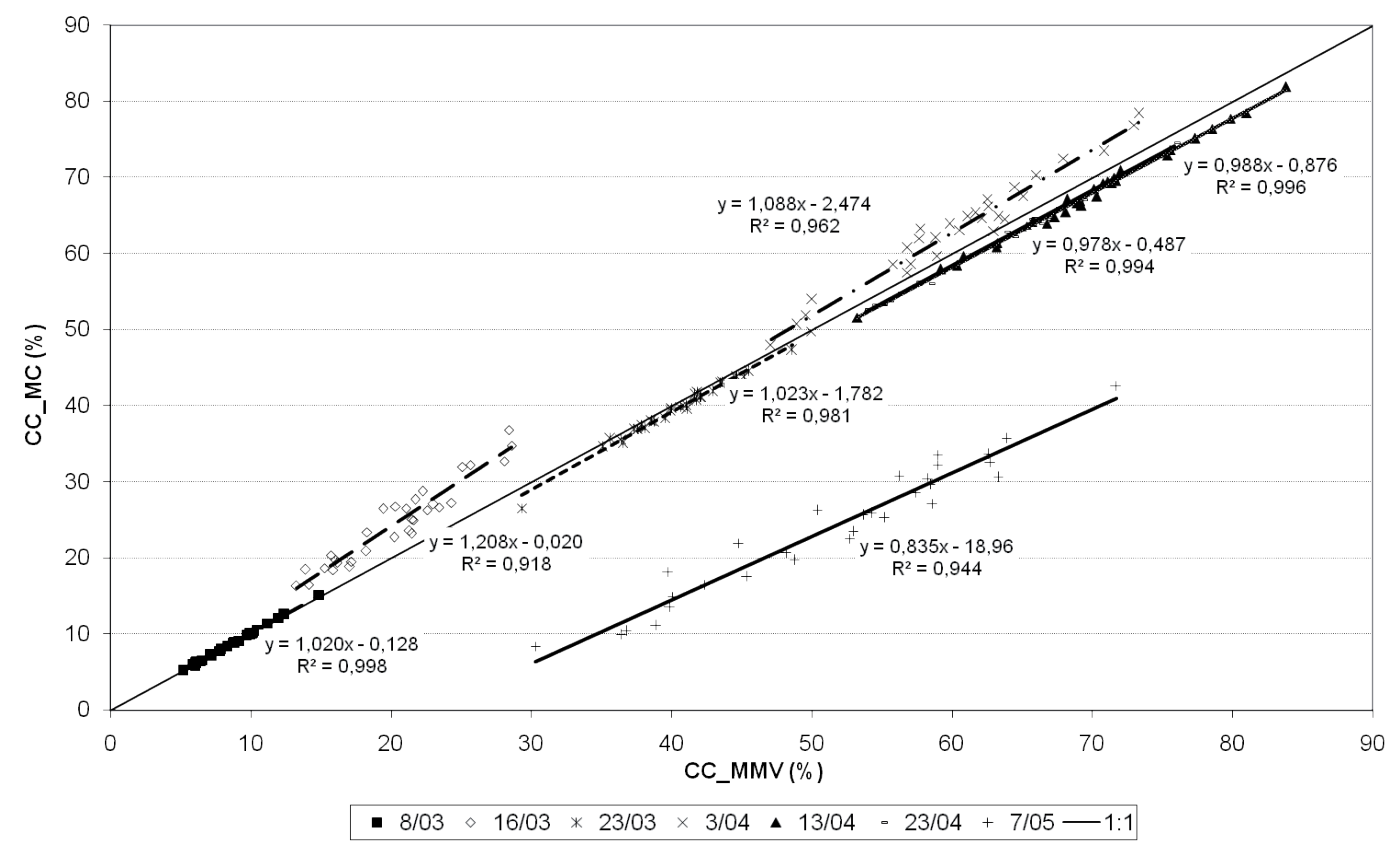

(A)

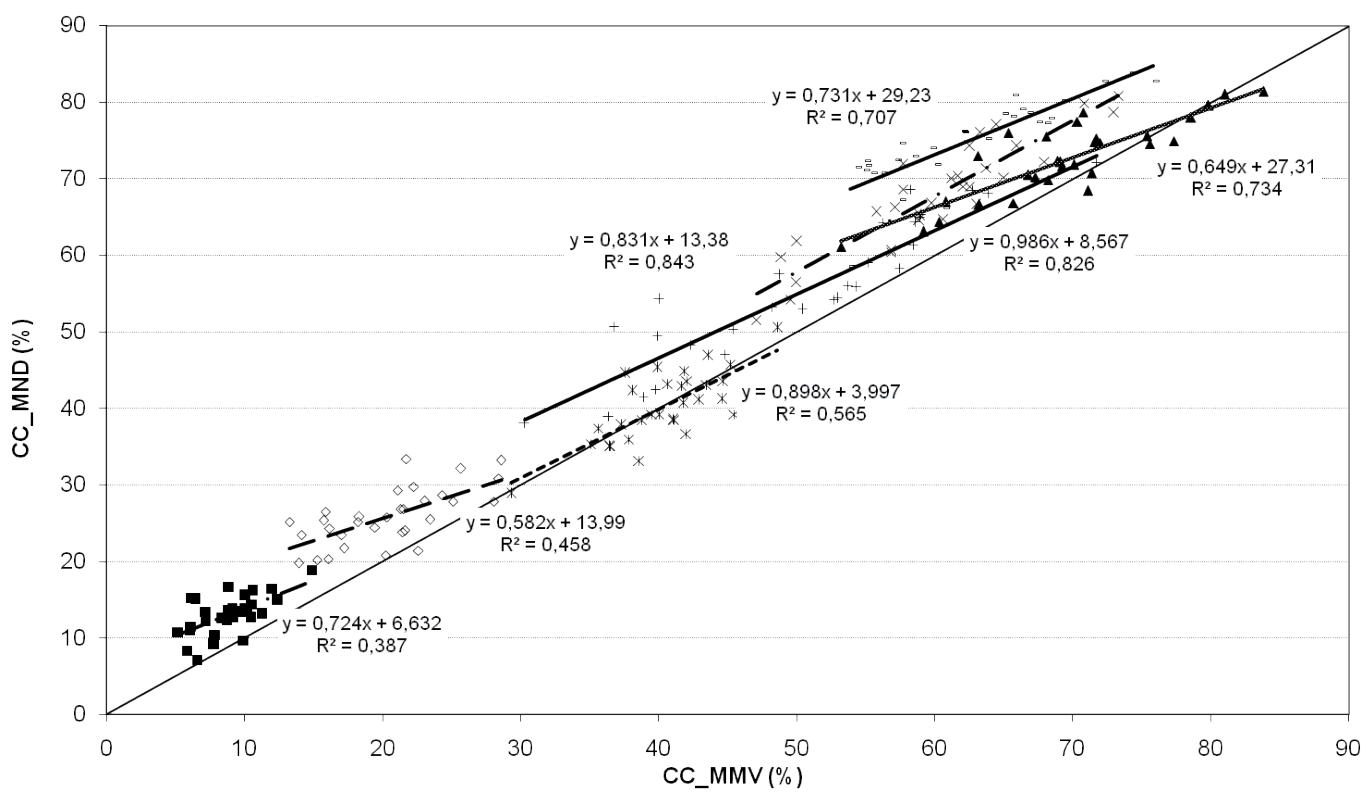

- $8 / 03 \diamond 16 / 03 * 23 / 03 \times 3 / 04 \times 13 / 04-23 / 04+7 / 05-1: 1$

(B)

Figura 1. Relación entre la cobertura del cultivo obtenida con el método del cociente (CC MC) y el método de máxima verosimilitud (CC MMV) (A) y entre el método que utiliza el NDVI (CC MND) y el método de máxima verosimilitud (CC MMV) (B), para distintas fechas durānte el ciclo de papa tardía 2012 en Córdoba, Argentina.

vado en 2008 bajo condiciones geográficas, ambientales y de cultivo semejantes (de la Casa et al., 2010), cuando la escena presenta abundante material amarillento y caído sobre el suelo al final del ciclo, el MC produce valores de la CC sistemática- mente menores a los de MMV. Con base en la información complementaria que se obtiene del NDVI, al considerar el nir, y de acuerdo a la similitud de los resultados que para la misma fecha se observa con el MMV, se pone de manifiesto que el uso de 
MC a partir de las bandas del visible sería apropiado sólo hasta que el cultivo alcanza cobertura máxima o hasta el comienzo de la senescencia del cultivo, en este caso con resultados prácticamente semejantes.

Cabe destacar que en 2012 la CC alcanzó niveles máximos sólo ligeramente superiores a $80 \%$, a pesar de la fertilización recibida, lo que redundó en valores de productividad más reducidos respecto a otros ciclos de producción (de la Casa et al., 2012). Para mostrar la variación de CC a lo largo del ciclo de acuerdo a las diferentes condiciones de fertilización, la Figura 2 presenta los valores medios para cada tratamiento de acuerdo a las estimaciones de CC mediante MC y MND.

La prueba de $t$ de la diferencia de medias apareadas para los valores de $\mathrm{CC}$, estimados tanto a partir de MC como de MND, presenta sólo valores significativos en la primera (8/03) y última $(7 / 05)$ de las fechas de muestreo, como así también cuando el análisis comprende a la totalidad del ciclo (Tabla 1). Este comportamiento se justifica ya que el cultivo no fue fertilizado a la plantación en el sector $\mathrm{NF}$, en tanto todo el lote se fertilizó posteriormente el 11/03, lo que repuso en general la condición de fertilidad. La diferencia de la CC al final del ciclo es probable que corresponda a la menor disponibilidad de $\mathrm{N}$ en el tratamiento NF, cuando hacia el final del ciclo el cultivo agotó anticipadamente el nutriente.

Como muestra la Figura 2, las estimaciones de CC que produce MND comienzan a ser mayores a MC aproximadamente a partir de los 45 días desde la plantación, pero estas diferencias recién resultan significativas el día 83 (Tabla 1). En esta última fecha también se evidencian diferencias significativas entre los tratamientos con fertilización y sin ésta, lo que avala el empleo de ambos medios digitales (cámaras convencional y modificada) a efectos de evaluar la condición de estrés nutricional.

\section{Productividad y calidad}

Tanto la deficiencia como el exceso de nitrógeno afectan la duración del ciclo del cultivo de papa $y$, en consecuencia, el rendimiento de tubérculos. La elevada disponibilidad de nitrógeno puede prolongar el crecimiento vegetativo, retrasar el inicio de tuberización y reducir el rendimiento (Saluzzo et al., 1999), como así también disminuir el porcentaje de materia seca de los tubérculos (Cieslik, 1997). Por su parte, el déficit de nitrógeno puede provocar una senescencia prematura de la parte aérea del cultivo debido a la translocación del nitrógeno desde las hojas hacia los tubérculos (Kleinkopf et al., 1981). Esta merma de la disponibilidad de $\mathrm{N}$

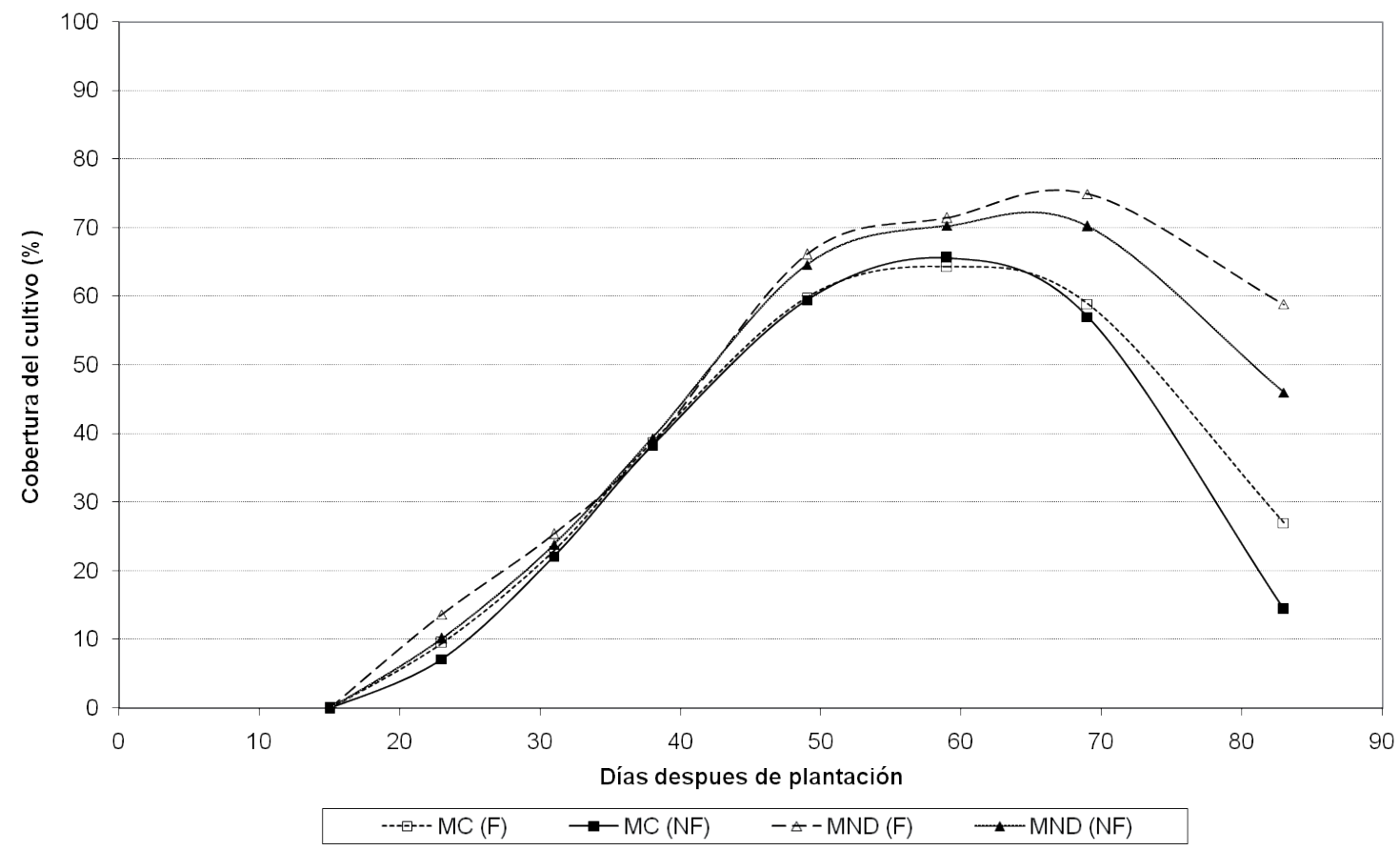

Figura 2. Valores medios de la cobertura del cultivo de papa obtenidos a partir de MC y MND de acuerdo a los días después de plantación para ambos tratamientos en 2012. 
Tabla 1. Valores de $t$ y probabilidad de la diferencia de medias apareadas $(n=9)$ de la cobertura del cultivo de papa estimados por el método del cociente (MC) y el método del NDVI (MND) para distintas condiciones de fertilización (F y NF) en 2012.

\begin{tabular}{|c|c|c|c|c|c|c|c|c|c|}
\hline \multirow{3}{*}{ Fecha } & \multirow{3}{*}{$n$} & \multicolumn{4}{|c|}{$\mathrm{MC}$} & \multicolumn{4}{|c|}{ MND } \\
\hline & & $\mathrm{F}$ & NF & $t$ & $P(T<=t)$ & $\mathrm{F}$ & NF & $t$ & $P(T<=t)$ \\
\hline & & \multicolumn{2}{|c|}{$(\%)$} & & & \multicolumn{2}{|c|}{$(\%)$} & & \\
\hline 08/03/2012 & 9 & 9,5 & 7,2 & 2,7971 & 0,0233 & 13,7 & 10,2 & 4,7301 & 0,0015 \\
\hline $16 / 03 / 2012$ & 9 & 23,2 & 22,1 & 0,6544 & 0,5312 & 25,3 & 23,9 & 1,4877 & 0,1751 \\
\hline 23/03/2012 & 9 & 38,8 & 38,2 & 0,4035 & 0,6971 & 38,5 & 39,4 & $-0,8345$ & 0,4282 \\
\hline 03/04/2012 & 9 & 59,9 & 59,5 & 0,1341 & 0,8967 & 66,2 & 64,7 & 0,5402 & 0,6038 \\
\hline $13 / 04 / 2012$ & 9 & 64,3 & 65,6 & $-0,4976$ & 0,6321 & 71,5 & 70,3 & 0,6231 & 0,5506 \\
\hline $23 / 04 / 2012$ & 9 & 58,9 & 57,1 & 0,7244 & 0,4894 & 75,0 & 70,3 & 1,8085 & 0,1081 \\
\hline $07 / 05 / 2012$ & 9 & 27,0 & 14,4 & 5,2880 & 0,0007 & 58,9 & 46,0 & 7,0957 & 0,0001 \\
\hline $8 / 3$ al $7 / 5$ & 63 & 40,2 & 37,7 & 2,6794 & 0,0094 & 49,9 & 43,5 & 3,9588 & 0,0002 \\
\hline
\end{tabular}

Diferencia significativas en negrita y cursiva.

explicaría la disminución acentuada que manifestó la CC de papa durante la parte final del ciclo, principalmente en el tratamiento NF.

Respecto a la producción, el sector F presentó mayor rendimiento total alcanzando una diferencia significativa con el tratamiento NF, como muestra la Tabla 2. Esta diferencia que produjo la menor disponibilidad de $\mathrm{N}$ se proyecta también en términos cualitativos, ya que ocasionó un cambio en el tamaño de los tubérculos. En la Tabla 2 se observa que la mayor fertilización incrementó la contribución de los tubérculos de tamaño grande, en tanto se reduce la proporción de los más pequeños, mientras que resulta indiferente estadísticamente el cambio en los tubérculos medianos. Este efecto que promueve la fertilización tiene importancia económica, localmente al menos, ya que la papa de mayor tamaño es preferida en la comercialización, además de que muchos de los tubérculos más pequeños no son levantados durante la cosecha.

Tabla 2. Valores de $t$ y probabilidad de la diferencia de medias apareadas $(n=12)$ del rendimiento final (total y clasificados por tamaño) del cultivo de papa para distintas condiciones de fertilización (F y NF) en 2012.

\begin{tabular}{|c|c|c|c|c|c|}
\hline & $\mathrm{n}$ & $\mathrm{F}$ & NF & $\mathrm{t}$ & $\mathrm{P}(\mathrm{T}<=\mathrm{t})$ \\
\hline & & \multicolumn{2}{|c|}{ Mg PF ha-1 } & & \\
\hline Total & 12 & 28,1 & 24,2 & 3,1226 & 0,0097 \\
\hline $\begin{array}{l}\text { Tubérculos } \\
\text { grandes }\end{array}$ & 12 & 16,6 & 10,7 & 4,3498 & 0,0011 \\
\hline $\begin{array}{l}\text { Tubérculos } \\
\text { medianos }\end{array}$ & 12 & 7,2 & 7,4 & $-0,2175$ & 0,8318 \\
\hline $\begin{array}{l}\text { Tubérculos } \\
\text { chicos }\end{array}$ & 12 & 4,2 & 6,1 & $-2,2119$ & 0,0491 \\
\hline
\end{tabular}

\section{Año 2013}

\section{Estimación de la cobertura}

La Figura 3 presenta la relación entre los valores de CC estimados alternativamente a partir de los métodos MC y MND para el conjunto completo de imágenes obtenidas del ensayo en 2013, discriminando en este caso los resultados anteriores y posteriores al 3/05, para representar las condiciones del canopeo en las etapas creciente y decreciente, respectivamente, de su variación durante el ciclo de cultivo.

La recta de ajuste lineal está prácticamente superpuesta a la función de identidad durante la etapa creciente del ciclo de desarrollo de la cobertura de papa este año. Además, los valores presentan menor dispersión en la etapa decreciente, particularmente hacia el final del ciclo, y la recta de ajuste tiende a diferenciarse, resultando sistemáticamente mayores los valores de CC que se estiman a partir del NDVI. No obstante, las imágenes obtenidas con la cámara convencional y las estimaciones de la cobertura a partir del método del cociente producen resultados muy similares a los que se alcanzan con la cámara modificada para calcular el NDVI, con mayores diferencias hacia la finalización del ciclo. Coincidente con Li et al. (2010) y Jia et al. (2016), este resultado avala el empleo de cámaras digitales convencionales de bajo costo, como así también la utilización de imágenes en un formato de baja resolución para realizar el monitoreo de la CC en papa. 


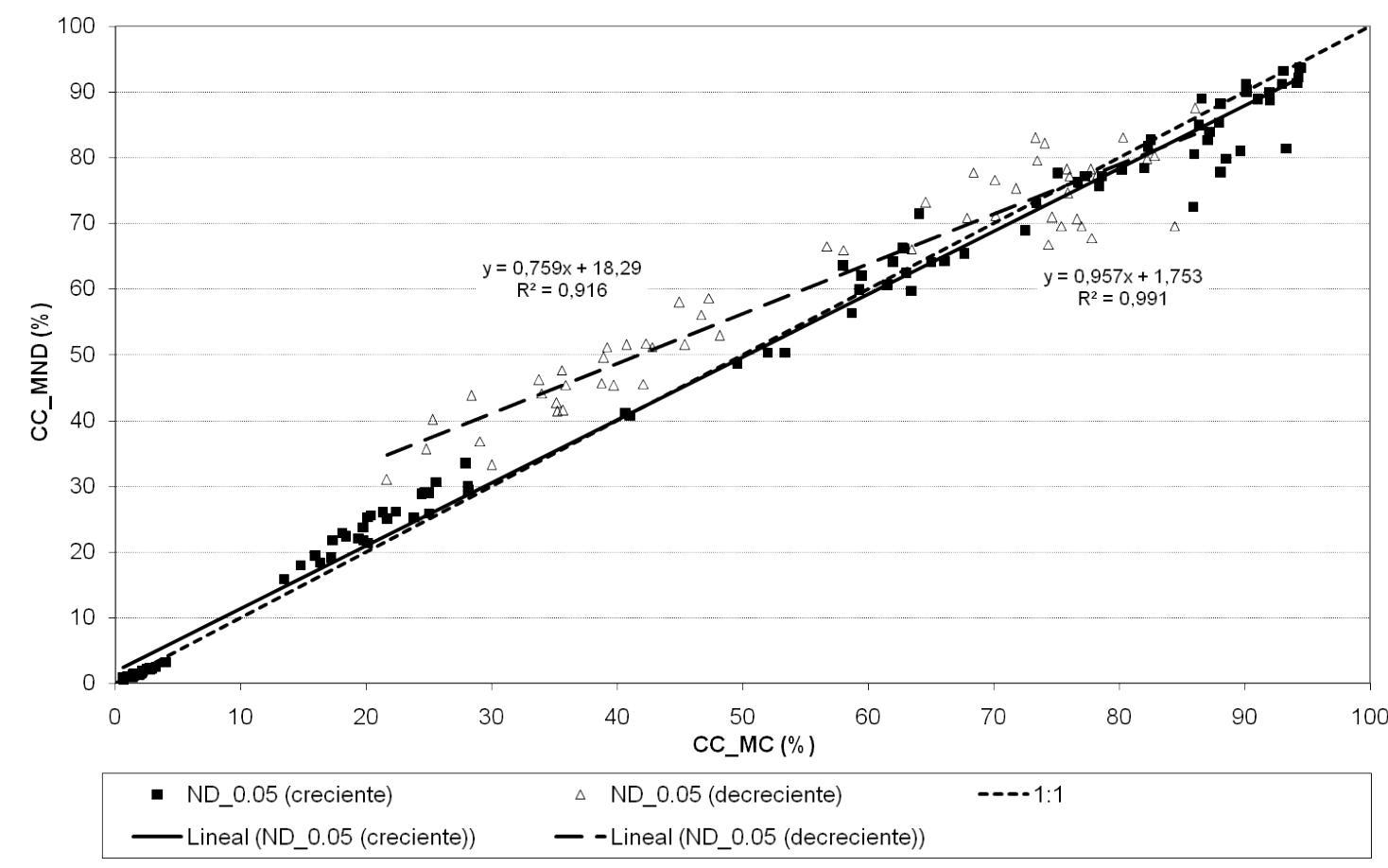

Figura 3. Relación entre la cobertura del cultivo obtenida con los métodos del cociente (CC MC) y el que utiliza el NDVI (CC MND), discriminando entre los valores crecientes y decrecientes durante el ciclo de papa 2013.

\section{Evaluación del contenido de $\mathrm{N}$ del suelo du- rante el ensayo}

A diferencia de 2012, y para disponer de información más completa sobre la disponibilidad de $\mathrm{N}$, durante el ensayo de 2013 se realizó la determi-

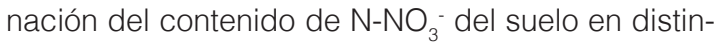
tas fechas, que arrojaron los valores que muestra la Figura 4. Después de la fertilización realizada a la plantación del cultivo, para caracterizar el tratamiento comercial se asignó la parcela que el 1/03 arrojó el contenido de $\mathrm{N}_{-} \mathrm{NO}_{3}^{-}$más bajo (6 ppm). Posterior a la refertilización y el agregado adicional de $\mathrm{N}$, el 27/03 se observó un incremento en el contenido de $\mathrm{N}^{-\mathrm{NO}_{3}-}$ en todos los tratamientos, y resultó menor el aumento (sin superar las 10 ppm) de la parcela (tratamiento comercial) que no recibió agregado adicional de N. En ella, las determinaciones posteriores arrojan valores de $\mathrm{N}_{-} \mathrm{NO}_{3}$ - de alrededor de 5 ppm, contenido que puede ser considerado inferior al nivel requerido por el cultivo (Westermann \& Kleinkopf, 1985; Feibert et al., 1998).

En los tratamientos que recibieron fertilización nitrogenada adicional, esta condición queda reflejada el 27/03 por el incremento hasta 20 ppm del contenido de $\mathrm{N}_{-} \mathrm{NO}_{3}$, sin mostrar diferencias entre las tres parcelas, a pesar de recibir dosis distintas de fertilizante. Las mediciones del mes de abril muestran una disminución acentuada del $\mathrm{N}$ en todas las parcelas, supeditado al intenso consumo del cultivo durante el periodo de máxima CC, que ocurrió entre 60 y 70 días después de plantación y alcanzó ese año valores mayores a 90\% (Figura 5). Por último, la medición al final del ciclo presenta niveles de $\mathrm{N}$ otra vez en aumento, siendo particularmente notable las 35 ppm del tratamiento con 150 $\mathrm{kg} \mathrm{N} \mathrm{ha}^{-1}$ adicionales. Este comportamiento puede justificarse como resultado de una lenta disponibilidad del fertilizante, debido quizas a que se fertilizó aproximadamente 2 meses antes del final del ciclo del cultivo, con la merma del consumo por parte del cultivo debido a su avanzado estado fenológico.

\section{Efecto de la fertilización sobre CC}

A fin de analizar la influencia que tuvo la fertilización adicional con $\mathrm{N}$ sobre el desarrollo de CC, la Figura 5 presenta los valores de CC a lo largo del ciclo de papa, estimados a partir de las imágenes de ambas cámaras y los procedimientos digitales usados respectivamente: MC y MND.

La Figura 5 muestra que la emergencia y establecimiento del cultivo de papa en 2013 se retrasó, alcanzando una cobertura incipiente recién después de 20 días de la plantación, cuando en pro- 
medio la etapa de plantación a emergencia se prolonga en esta región entre 10 y 15 días (Ovando et al., 2006). También se pone de manifiesto un efecto positivo de la fertilización en el crecimiento de CC que, cuando se consideran las estimaciones de CC a partir de MC (Figura 5 A), es más notable a partir de los 40 días después de plantación y hacia la finalización del ciclo. Las determinaciones de CC con MND (Figura 5, B), en cambio, aunque sólo con ligeras diferencias respecto al otro procedimiento, muestran un menor desarrollo de CC para

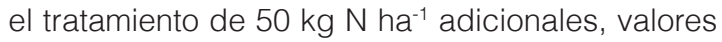
que se mantienen por debajo del comercial duran- te la mayor parte del ciclo. Haciendo esta salvedad a favor de la mayor sensibilidad del procedimiento que emplea el NDVI para diferenciar el material foliar activo del suelo, los valores de CC resultan en general más elevados en los tratamientos que recibieron 100 y $150 \mathrm{~kg} \mathrm{~N}^{-1}$ adicionales respecto al tratamiento comercial; estas diferencias son reconocibles ya sea que la estimación provenga de MC como de MND.

La diferencia de CC entre tratamientos es significativa en términos estadísticos, sólo en la fecha del último muestreo al final del ciclo y cuando se considera el ciclo completo; lo mismo si CC se ob-

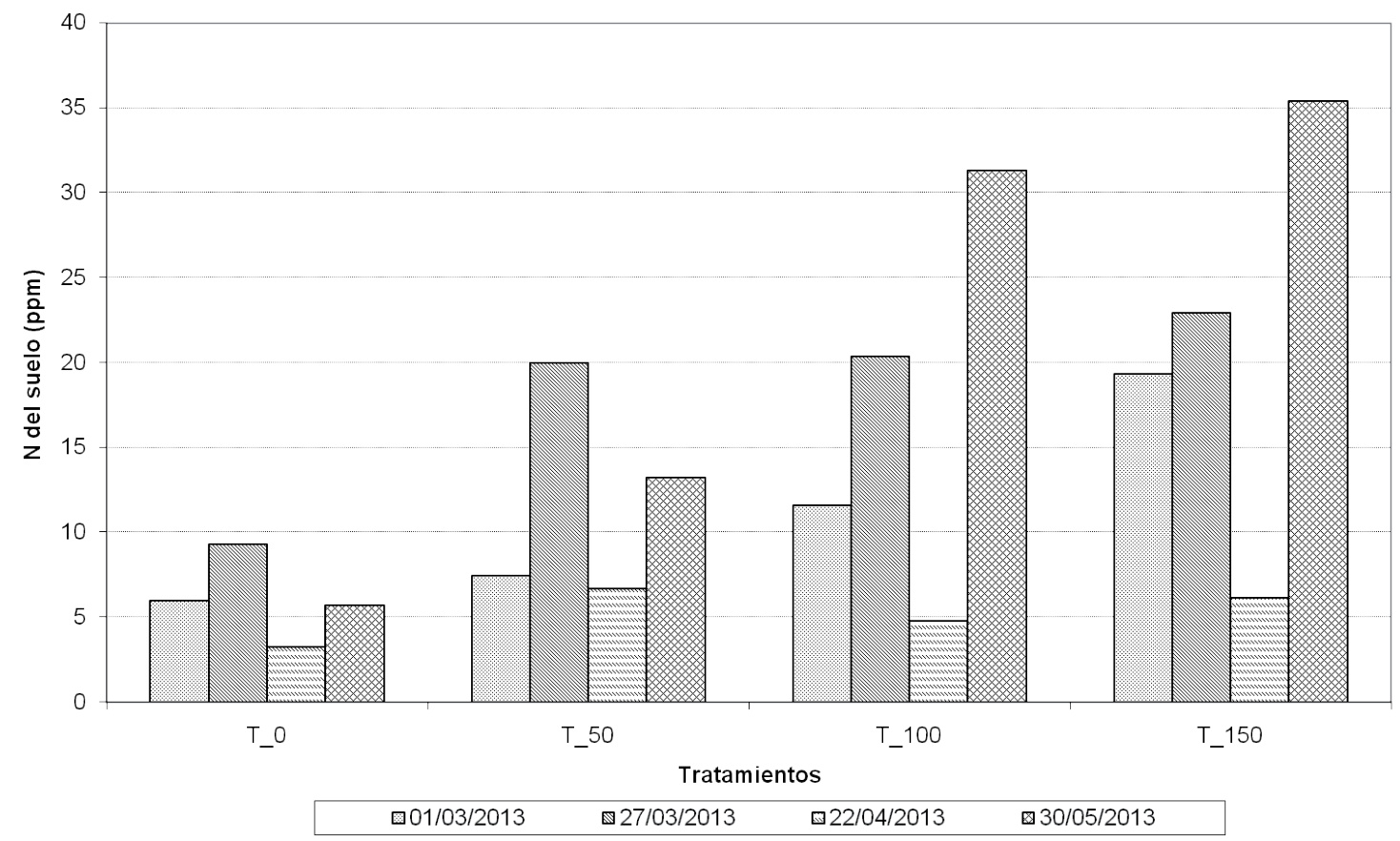

Figura 4. Variación del contenido de $\mathrm{N}$ del suelo en los distintos tratamientos del ensayo de fertilización del cultivo de papa en 2013.

Tabla 3. Valores de $t$ y probabilidad de la diferencia de medias apareadas $(n=7)$ del rendimiento final (total y de los tubérculos clasificados por tamaño) del cultivo de papa para distintas condiciones de fertilización (NF: sin agregado de N adicional, y con 50,100 y $150 \mathrm{~kg}$ de $\mathrm{N} \mathrm{ha}^{-1}$ adicionales) en 2013. La diferencia para cada nivel de fertilización se establece con respecto a NF.

\begin{tabular}{|c|c|c|c|c|c|c|c|c|c|c|}
\hline \multirow{3}{*}{ Tratamiento } & \multicolumn{4}{|c|}{ Rendimiento } & \multicolumn{6}{|c|}{ Prueba de medias apareadas } \\
\hline & \multirow[t]{2}{*}{ NF } & 50 & 100 & 150 & \multicolumn{2}{|c|}{50} & \multicolumn{2}{|c|}{100} & \multicolumn{2}{|c|}{150} \\
\hline & & \multicolumn{2}{|c|}{ Mg PF ha-1 } & & $\mathrm{t}$ & $\mathrm{P}(\mathrm{T}<=\mathrm{t})$ & $\mathrm{t}$ & $\mathrm{P}(\mathrm{T}<=\mathrm{t})$ & $\mathrm{t}$ & $\mathrm{P}(\mathrm{T}<=\mathrm{t})$ \\
\hline Total & 30,1 & 27,8 & 36,2 & 32,8 & 0,6107 & 0,5369 & $-2,4569$ & 0,0493 & $-0,7363$ & 0,4893 \\
\hline $\begin{array}{l}\text { Tubérculos } \\
\text { grandes }\end{array}$ & 19,0 & 19,1 & 21,1 & 23,2 & $-0,0240$ & 0,9817 & $-0,4433$ & 0,6731 & $-1,3272$ & 0,2327 \\
\hline $\begin{array}{l}\text { Tubérculos } \\
\text { medianos }\end{array}$ & 8,4 & 5,6 & 9,1 & 5,6 & 1,4273 & 0,2034 & $-0,3435$ & 0,7429 & 1,6820 & 0,1436 \\
\hline $\begin{array}{l}\text { Tubérculos } \\
\text { chicos }\end{array}$ & 2,6 & 3,1 & 3,9 & 4,0 & $-0,3768$ & 0,7193 & $-0,6943$ & 0,5134 & $-1,2484$ & 0,2584 \\
\hline
\end{tabular}



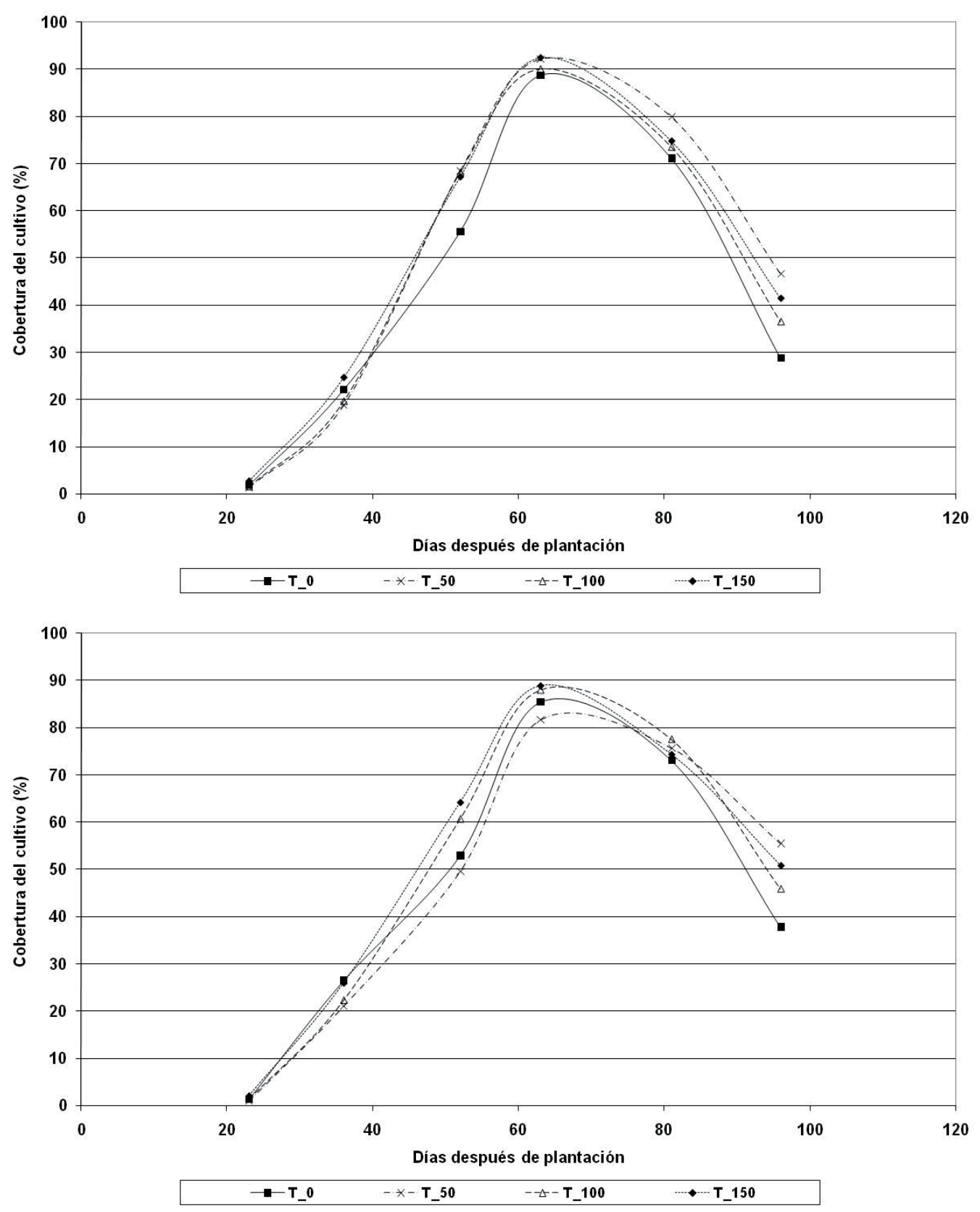

(A)

(B)

Figura 5. Variación de la cobertura del cultivo de papa estimada con MC (A) y MND (B) respecto a los días después de plantación para los distintos tratamientos en 2013: control (T_0), 50 (T_50), 100 (T_100) y 150 (T_150) kg N ha-1 adicionales.

tiene a partir de MC con una cámara convencional, como de MND usando la cámara modificada. Si bien las diferencias entre tratamientos en 2013 son menos pronunciadas que lo observado el año anterior, se corrobora que la fertilización adicional prolonga por más tiempo una CC de mayor envergadura, y le permite al cultivo de papa alcanzar un mayor potencial de rendimiento (de la Casa et al., 2012; 2013).

\section{Productividad y calidad}

La Tabla 3 muestra los resultados arrojados en el análisis estadístico de la cosecha 2013. Sólo se presentó significativa $(P<0,05)$ la diferencia del rendimiento total entre el tratamiento comercial y el que recibió $100 \mathrm{~kg}$ de $\mathrm{N} \mathrm{ha}^{-1}$ adicionales, sin que esta diferencia se traslade a ninguna categoría de tamaño de los tubérculos en particular, como suce- 
dió en 2012. De acuerdo a la Figura 4, el contenido de $\mathrm{N}_{-} \mathrm{NO}_{3}$ - del suelo del tratamiento comercial se presentó siempre por debajo del requerimiento, en tanto el 24/4, durante la etapa de CC máximo, el contenido resulta también insuficiente para todos los tratamientos. Estas limitaciones de la disponibilidad de $\mathrm{N}$ explican en buena medida que las diferencias de producción entre los tratamientos en 2013 sólo se expresen parcialmente.

La menor productividad del tratamiento con 50 $\mathrm{kg}$ de $\mathrm{N} \mathrm{ha}^{-1}$ adicionales respecto al rendimiento comercial se explica a partir del menor desarrollo alcanzado por la CC, que se detectó sólo a partir de MND con la cámara modificada durante la etapa creciente del ciclo. La falta de respuesta en el rendimiento del tratamiento con $150 \mathrm{~kg}$ de $\mathrm{N} \mathrm{ha}^{-1}$ adicionales, por su parte, sugiere que el valor de CC no puede interpretarse en términos absolutos respecto de la productividad del cultivo y que, a los efectos de alcanzar mayor potencial predictivo, será necesario considerar otros elementos de la imagen, como la intensidad del color (ligada a la concentración de clorofila), para diferenciar las condiciones de productividad bajo distintas tratamientos de fertilización.

Como Li et al. (2010) señalan, las bandas verde y roja de las cámaras digitales de uso doméstico, resultan apropiadas para determinar la proporción de suelo cubierto por el follaje y, asimismo, por su adecuada correlación con la biomasa aérea, pueden ser útiles a los efectos de establecer el requerimiento de fertilización con N. Sin embargo, otros dispositivos como GreenSeeker $\AA$ y el Yara $\mathrm{N}-\mathrm{Sensor}$ presentan mayor sensibilidad para detectar el estado de la vegetación que el monitoreo de CC a partir de los canales visibles. De igual forma, en un experimento de fertilización en maíz, Hunt et al. (2005) no detectaron diferencias en el estado de $\mathrm{N}$ a partir del indicador NGRDI (elaborado con los canales verde y rojo), aunque las áreas con escaso $\mathrm{N}$ eran claramente visibles al final de la estación a partir de fotografías digitales. En un análisis en el que se usó el modelo de transferencia radiativa del canopeo SAIL (scattering of arbitrarily inclined leaves), verificaron que NGRDI es un indicador sensible a la biomasa antes del cierre del canopeo, pero que las variaciones en la concentración de clorofila no serían detectables por este medio.

\section{CONCLUSIONES}

Ambas cámaras utilizadas, tanto la comercial que produce imágenes a color, como la modificada para obtener imágenes de NDVI, como así también los procedimientos digitales de clasificación propuestos: método de máxima verosimilitud (MMV), método del cociente de bandas (MC) y el que utiliza el NDVI (MND), se presentan apropiados para estimar CC en papa. El MC sólo reduce su sensibilidad para determinar CC cuando el cultivo de papa entra en la etapa de senescencia, aunque esto fue más evidente en 2012.

La diferencia en la fertilización y la consecuente reducción del $\mathrm{N}$ disponible, produjo una disminución anticipada de CC al final del ciclo que redundó en una menor productividad del cultivo de papa en 2012. Además, la diferencia de fertilización también comprometió ese año la calidad comercial al reducir la proporción de los tubérculos de mayor tamaño. En 2013, probablemente por limitaciones en la disponibilidad de N, las diferencias de producción entre los tratamientos sólo se expresaron parcialmente y no se presentó ninguna diferencia de carácter cualitativa respecto al tamaño de los tubérculos.

Los resultados de 2013 limitan el valor de CC como parámetro absoluto para determinar el comportamiento productivo del cultivo de papa y justifican la evaluación de otras características de la imagen a los efectos de producir herramientas más generalizadas destinadas a predecir el rendimiento.

\section{AGRADECIMIENTO}

Los autores agradecen a la Secretaría de Ciencia y Técnica de la Universidad Nacional de Córdoba por el apoyo económico recibido.

\section{BIBLIOGRAFÍA}

Adamsen, F.J.; P.J. Pinter Jr., E.M. Barnes, R.L. La Morte, G.W. Wall, S.W. Leavitt and B.A. Kimball, 1999. Measuring wheat senescence with a digital camera. Crop Sci. 39:719-724.

Adamsen, F.J.; T.A. Coffelt, J.M. Nelson, E.M. Barnes and R.C. Rice, 2000. Method for using images from a color digital camera to estimate flower number. Crop Sci. 40:704-709.

Caldiz, D.O., 2006. Producción, cosecha y almacenamiento de papa en la Argentina. 226 p. BASF Argentina-McCain, Buenos Aires, Argentina.

Cieslik, E., 1997. Effect of the levels of nitrates and nitrites on the nutritional and sensory quality of potato tubers. Hygiene and Nutrition in Foodservice and Catering 1:225-230.

de la Casa, A.; G. Ovando, L. Bressanini, Á. Rodríguez y J. Martínez, 2007. Uso del índice de área foliar y 
del porcentaje de cobertura del suelo para estimar la radiación interceptada en papa. Agricultura Técnica (Chile) 67(1):78-85.

de la Casa, A.; G. Ovando, L. Bressanini, Á. Rodríguez y J. Martínez, 2010. Determinación de la fracción de suelo cubierta con el follaje de papa a partir del cociente entre bandas de fotografías digitales. Actas de la XIII Reunión Argentina y VI Latinoamericana de Agrometeorología. Bahía Blanca, Buenos Aires, Argentina.160-161 pp.

de la Casa, A.; G. Ovando, L. Bressanini, J. Martínez y A. Rodríguez, 2012. Predicción de la variabilidad del rendimiento de papa a partir de la cobertura del follaje. Agriscientia 29:15-24.

de la Casa, A.; G. Ovando, L. Bressanini and J. Martínez, 2013. Aquacrop model calibration in potato and its use to estimate yield variability under field conditions. Atmospheric and Climate Sciences 2013(3):397-407.

Eastman, J.R.; S. Crema, H. Zhu, J. Toledano and H. Jiang, 2005. In-process classification assessment of remotely sensed imagery. Geocarto International 20(4):33-43.

Ewing, R.P. and R. Horton. 1999. Quantitative color image analysis of agronomic images. Agron. J. 91:148-153.

Feibert, E.B.G.; C.C. Shock and L.D. Saunders, 1998. Nitrogen fertilizer requirements of potatoes using carefully scheduled sprinkler irrigation. HortScience 33:262-265.

Giletto, C.M.; C. Díaz, J.E. Rattín, H.E. Echeverría and D.O. Caldiz, 2010. Green index to estimate crop nitrogen status in potato processing varieties. Chilean Journal of Agricultural Research 70(1):142-149.

Gitelson, A.A.; Y.J. Kaufman, R. Stark and D. Rundquist, 2002. Novel algorithms for remote estimation of vegetation fraction. Remote Sens. Environ. 80:76-87.

Gitelson, A.A., Y. Peng and K.F. Huemmrich, 2014. Relationship between fraction of radiation absorbed by photosynthesizing maize and soybean canopies and NDVI from remotely sensed data taken at close range and from MODIS $250 \mathrm{~m}$ resolution data. Remote Sens. Environ. 147:108-120.

Hatfield, J.L.; A.A. Gitelson, J.S. Schepers and C. L. Walthall, 2008. Application of spectral remote sensing for agronomic decisions. Agron. J. 100, S117-S131.

Hunt, E.R.; M. Cavigelli, C.S.T Daughtry, J.E. McMurtrey III and C.L. Walthall, 2005. Evaluation of digital photography from model aircraft for remote sensing of crop biomass and nitrogen status. Precision Agriculture 6:359-378

Hunt, E.R.; W.D. Hively, S.J. Fujikawa, D.S. Linden, C.S. Daughtry and G. McCarty, 2010. Acquisition of NIRgreen-blue digital photographs from unmanned aircraft for crop monitoring. Remote Sensing 2(1):290-305.
Jannoura, R.; K. Brinkmann, D. Uteau, C. Bruns and R.G. Joergensen, 2015. Monitoring of crop biomass using true colour aerial photographs taken from a remote controlled hexacopter. Biosystems Engineering 129:341-351.

Jia, K.; S. Liang, X. Gu, F. Baret, X. Wei, X. Wang, Y. Yao, L. Yang and Y. Li, 2016. Fractional vegetation cover estimation algorithm for Chinese GF-1 wide field view data. Remote Sens. Environ. 177:184-191.

Johnson, L.F. and T.J. Trout, 2012. Satellite NDVI assisted monitoring of vegetable crop evapotranspiration in California's San Joaquin Valley. Remote Sensing $4(2): 439-455$.

Kenney, W.A., 1987. A method for estimating windbreak porosity using digitized photographic silhouettes. Agricultural and Forest Meteorology 39:91-94.

Kleinkopf, G.E.; D.T. Westermann and R.B. Dwelle, 1981. Dry matter production and nitrogen utilization by six potato cultivars. Agronomy Journal 73: 799-802.

Li, Y.; D. Chen, C.N. Walker and J.F. Angus, 2010. Estimating the nitrogen status of crops using a digital camera. Field Crops Research 118:221-227.

Meyer, G.E. and J.C. Neto, 2008. Verification of color vegetation indices for automated crop imaging applications. Computers and Electronics in Agriculture 63(2):282-293.

Muraoka, H.; H.M. Noda, S. Nagai, T. Motohka, T.M. Saitoh, K.N. Nasahara and N. Saigusa, 2012. Spectral vegetation indices as the indicator of canopy photosynthetic productivity in a deciduous broadleaf forest. Journal of Plant Ecology Doi: 10.1093/jpe/ rts037.

Ovando, G.; A. de la Casa y A. Rodríguez, 2006. Estimación de la longitud del brote de papa en el subperíodo plantación-emergencia utilizando diferentes métodos de cómputo del tiempo térmico. XI Reunión Argentina de Agrometeorología, Asociación Argentina de Agrometeorología. La Plata, Buenos Aires. 205-206 pp.

Purcell, L.C., 2000. Soybean canopy coverage and light interception measurement using digital imagery. Crop Sci. 40:834-837.

Raun, W.R.; J.B. Solie, R.K. Taylor, D.B. Arnall, C.J. Mack and D.E. Edmonds, 2008. Ramp calibration strip technology for determining midseason nitrogen rates in corn and wheat. Agron. J. 100:1088-1093.

Rouse, J.W.; R.H. Haas, J.A. Schell and D.W. Deering, 1973. Monitoring Vegetation Systems in the Great Plains with ERTS. Third ERTS Symposium, NASA SP351 I: 309-317.

Saluzzo, A.; H. Echeverría, F.H. Andrade and M. Huarte, 1999. Nitrogen nutrition of potato cultivars differing in maturity. J. Agron. \& Crop Sci. 183:157-165

Steduto, P.; T.C. Hsiao, D. Raes and E. Fereres, 2009. 
AquaCrop-The FAO crop model for predicting yield response to water: I. Concepts and underlying principles. Agron. J. 101:426-437.

Von Bueren, S.K.; A. Burkart, A. Hueni, U. Rascher, M.P. Tuohy and I.J. Yule, 2015. Deploying four optical UAVbased sensors over grassland: challenges and limita- tions. Biogeosciences 12(1):163-175.

Wang, F.M.; J.F. Huang, Y.L. Tang and X.Z. Wang, 2007. New vegetation index and its application in estimating leaf area index of rice. Rice Science 14(3):195-203.

Westermann, D.T. and G.E. Kleinkopf, 1985. Nitrogen requirements of potatoes. Agron. J. 77:616-621. 